Patrick JAGORET ${ }^{1}$

Jacques KWESSEU 2, 3

Charly Anicet MESSIE2, 3

Isabelle MICHEL ${ }^{4}$

Éric MALÉZIEUX ${ }^{5}$

${ }^{1}$ Cirad

UMR System

2, place Viala

34060 Montpellier Cedex 2

France

2 Irad

Nkolbisson

BP 2572

Yaoundé

Cameroun

3 Université de Dschang

Fasa

BP 222

Dschang

Cameroun

${ }^{4}$ Montpellier Supagro-Institut des régions chaudes UMR Innovation

1101, avenue Agropolis BP 5098

34093 Montpellier Cedex 5

France

5 Cirad

UPR HortSys

TA B-103 / PS4

Boulevard de la Lironde 34398 Montpellier Cedex 5 France

\title{
Valeurs d'usage des ligneux utilisés en agroforesterie :
les cacaoyères du Centre-Cameroun utilisés en agroforesterie :
les cacaoyères du Centre-Cameroun
}

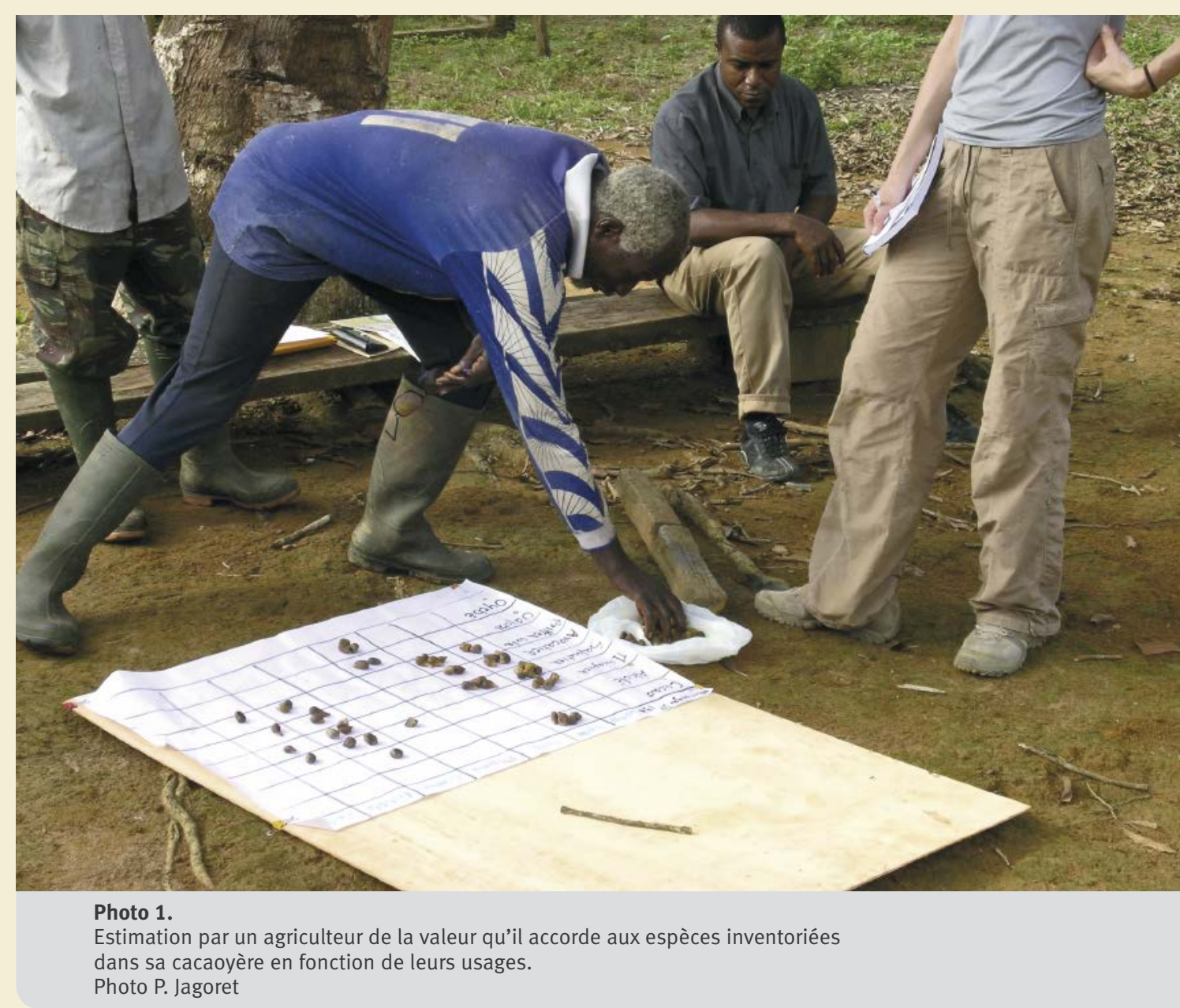

Photo 1.

Estimation par un agriculteur de la valeur qu'il accorde aux espèces inventoriées dans sa cacaoyère en fonction de leurs usages. Photo P. Jagoret 


\section{RÉSUMÉ}

\section{VALEURS D'USAGE DES LIGNEUX UTILISÉS EN AGROFORESTERIE : LES CACAOYĖRES DU CENTRE-CAMEROUN}

En zone tropicale humide, la valeur des systèmes agroforestiers pour les agriculteurs est souvent difficile à évaluer. À partir d'un inventaire floristique réalisé dans 14 cacaoyères agroforestières du Centre-Cameroun, l'article propose leur évaluation participative basée sur la méthode de distribution des cailloux mobilisée pour quantifier la valeur accordée par les agriculteurs à chaque espèce en fonction des usages attribués. Au total, 86 espèces ligneuses ont été inventoriées. Les cinq espèces les plus fréquentes ont été le safoutier, Dacryodes edulis, avec $18 \%$ des arbres inventoriés, l'avocatier, Persea americana, avec $11 \%$, le fraké, Terminalia superba, avec $10 \%$, le figuier, Ficus mucoso, avec $5 \%$, et Albizia adianthifolia, avec $3 \%$. Vingt-deux espèces (26\%) n'ont eu aucun usage pour les agriculteurs qui ont défini sept usages différents pour les autres espèces inventoriées. Parmi ces espèces, la valeur d'usage la plus élevée a été attribuée au cacaoyer (20\%). Ensuite, par ordre décroissant, les cinq espèces qui ont eu les valeurs d'usage les plus élevées ont été l'ayous, Triplochyton scleroxylon, le sapelli, Entandrophragma cylindricum, le safoutier, Dacryodes edulis, le palmier à huile, Elaeis guineensis, et le manguier, Mangifera indica. Le profil d'usage des cacaoyères a montré qu'elles sont principalement pilotées par les agriculteurs pour la vente de produits ligneux et non ligneux, l'autoconsommation de produits non ligneux et de produits médicinaux. Les résultats indiquent que les innovations techniques visant à améliorer les systèmes agroforestiers cacaoyers doivent tenir compte de leur complexité et de leur plurifonctionnalité.

Mots-clés : Theobroma cacao L., agroforesterie, connaissances locales, gestion des ressources naturelles, évaluation participative, méthode de distribution des cailloux, Cameroun.

\section{ABSTRACT}

\section{USE VALUES OF WOODY SPECIES USED IN AGROFORESTRY: COCOA AGROFORESTS IN CENTRAL CAMEROON}

In humid tropical regions, the value to farmers of agroforestry systems is often difficult to assess. Based on an inventory of plant species in 14 cocoa agroforests in central Cameroon, this study developed a participatory assessment using a PRA (Participatory Rural Appraisal) scoring system to quantify the value given by farmers to each species according to their uses. Altogether, 86 species were identified. The five species most commonly found were African plum, Dacryodes edulis at $18 \%$ of the trees identified, avocado, Persea americana at $11 \%$, limba, Terminalia superba at $10 \%$, the fig tree, Ficus mucoso at $5 \%$ and Albizia adianthifolia at 3\%. Twentytwo species (26\%) were not used at all by the farmers, who defined seven different uses for the other species. Among these, the highest use value was attributed to the cocoa tree $(20 \%)$. The next five tree species with high use values were, in decreasing order, the ayous, Triplochyton scleroxylon, sapelli, Entandrophragma cylindricum, African plum, Dacryodes edulis, oil palm, Elaeis guineensis, and mango, Mangifera indica. The use profile for cocoa agroforests showed that these are mainly managed by farmers for sales of timber and non-timber products and for home consumption of non-timber and medicinal products. These results show that technical innovations to improve cocoa agroforestry systems must take into account their multiple functions and their complexity.

Keywords: Theobroma cacao L., agroforestry, local knowledge, natural resource management, participatory assessment, PRA scoring system, Cameroon.

\section{VALORES DE USO DE ESPECIES LEÑOSAS UTILIZADAS EN AGROFORESTERÍA: LOS CACAOTALES DEL CENTRO DE CAMERÚN}

En las zonas tropicales húmedas, el valor de los sistemas agroforestales suele ser de difícil evaluación para los agricultores. A partir de un inventario florístico realizado en 14 cacaotales agroforestales de la región del Centro de Camerún, el artículo propone su evaluación participativa basada en el método de distribución de piedritas con las que los propios agricultores cuantifican el valor que otorgan a cada especie según sus usos. Se inventariaron un total de 86 especies leñosas. Las especies más frecuentes fueron atanga, Dacryodes edulis, con $18 \%$, aguacate, Persea americana, con $11 \%$, limba, Terminalia superba, con $10 \%$, higuera, Ficus mucoso, con $5 \%$ y Albizia adianthifolia, $3 \%$. Veintidós especies (26\%) no tenían ninguna utilidad para los agricultores, que habían definido siete usos diferentes para las demás especies inventariadas. El valor de uso más alto de estas especies se atribuyó al cacaotero (20\%); seguidamente, por orden decreciente, las cinco especies con valores de uso más altos fueron: obeche, Triplochiton scleroxylon, sapelli, Entandrophragma cylindricum, atanga, Dacryodes edulis, palma aceitera, Elaeis guineensis, y mango, Mangifera indica. El perfil de uso de los cacaoteros muestra que los agricultores los destinan principalmente para venta de productos madereros y no madereros, autoconsumo de productos no madereros y productos medicinales. Los resultados muestran que las innovaciones técnicas destinadas a mejorar los sistemas agroforestales cacaoteros deben tener en cuenta su complejidad y multifuncionalidad.

Palabras clave: Theobroma cacao L., agroforestería, conocimientos locales, manejo de recursos naturales, evaluación participativa, método de distribución de piedritas, Camerún. 


\section{Introduction}

Pays producteur de cacao depuis le début du XXe siècle, le Cameroun occupe aujourd'hui le cinquième rang mondial avec des exportations annuelles qui, depuis 2007, ont été en moyenne de 210000 tonnes (ICCO, 2012). La production est essentiellement issue de deux régions, le Sud-Ouest et le Centre-Sud, principal bassin de production où la cacaoculture repose en grande partie sur des cacaoyères agroforestières qui demeurent le socle de nombreuses exploitations en termes d'assolement et de revenu (Jagoret et al., 2011).

Ces cacaoyères constituent des peuplements plurispécifiques multistrates à haute valeur environnementale et sociale (Ruf et Schroth, 2004 ; Bisseleua et al., 2009). Nombreuses sont les espèces associées aux cacaoyers qui jouent également un rôle important dans le fonctionnement des exploitations. Plusieurs espèces, tout en fournissant un ombrage aux cacaoyers, procurent aux ménages une multitude de produits (fruits, bois, feuilles, écorces) commercialisés ou non, qui interviennent dans l'autosuffisance et l'équilibre alimentaire des familles, la pharmacopée, la construction des habitations et la trésorerie des exploitations (Sonwa et al., 2007).

Les travaux existants réalisés sur les systèmes agroforestiers cacaoyers du Centre-Sud du Cameroun ne permettent cependant pas leur évaluation globale. Ces systèmes, quelle que soit leur composante principale, demeurent en effet difficiles à évaluer en raison des problèmes méthodologiques que pose l'évaluation des espèces qui les composent, dont diffèrent les usages, la nature des produits récoltés ou des services rendus, et les cycles de développement (Nair, 1993 ; Huxley, 1999). Certaines espèces rendent plusieurs types de services, d'ordre écologique notamment,

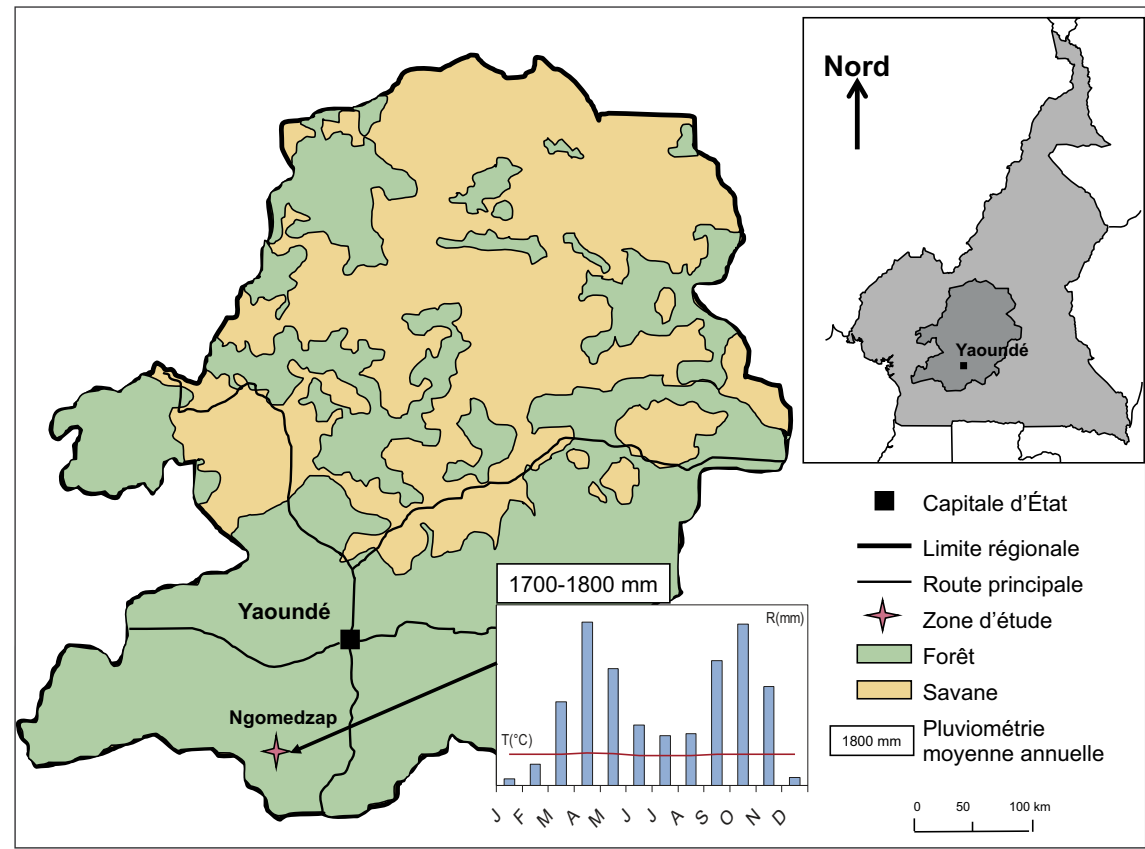

Figure 1.

Localisation de la région Centre et de la zone d'étude. qui s'avèrent difficilement quantifiables et posent la question de l'unité commune à retenir (Huxley, 1999). Sur le plan économique, une autre difficulté de l'évaluation est liée au fait que la plupart des espèces considérées sont pérennes, gérées sur le long terme, et constituent des peuplements hétérogènes en termes de structure et d'âge en raison des pratiques des agriculteurs (Torquebiau et al., 2002).

Afin de contourner ces difficultés, l'article propose une méthode d'évaluation participative des systèmes agroforestiers, basée sur la valeur d'usage relative attribuée par l'agriculteur aux différentes espèces. Cette méthode, inspirée de la méthode de distribution des cailloux mobilisée pour l'évaluation de paysages (Sheil et al., 2004), a été testée en 2009 dans 14 cacaoyères de la zone forestière du Centre-Cameroun.

\section{Matériels et méthode}

\section{Zone d'étude}

L'étude a été conduite en 2009 dans la région du Centre, dans la zone de Ngomedzap (département du Nyong et So’o) (figure 1). Cette zone, localisée au sud de Yaoundé, est située sur le plateau sud-camerounais, à une altitude de 600 à $800 \mathrm{~m}$. Le climat est chaud et humide, de régime bimodal, avec une température moyenne annuelle de $25^{\circ} \mathrm{C}$ et une pluviométrie moyenne annuelle de $1800 \mathrm{~mm}$. Il s'agit d'une zone forestière caractérisée par une végétation dominée par la forêt dense sempervirente et des sols ferrallitiques fortement désaturés. La densité de population y est de l'ordre de 37 hab. $/ \mathrm{km}^{2}$ (Santoir et Bopda, 1995).

\section{Dispositif expérimental}

L'étude a été conduite dans 14 cacaoyères agroforestières productives, âgées de 21 à 71 ans, et appartenant à 11 agriculteurs, un agriculteur pouvant gérer plusieurs cacaoyères.

\section{Estimation de la valeur d'usage des espèces}

Un inventaire des espèces ligneuses associées aux cacaoyers a été réalisé. Tous les arbres d'une hauteur supérieure à $1 \mathrm{~m}$ ont été inventoriés sur la surface totale de chaque cacaoyère. L'identification des espèces a été basée sur les noms vernaculaires en langue ewondo. Les correspondances avec les noms scientifiques ont été établies à partir des manuels d'identification de Vivien et Faure (1985), et Eyog Matig et al. (2006). Dans le même temps, les agriculteurs ont été questionnés sur l'origine de chaque espèce (spontanée et préservée ou introduite) et ses différents usages, y compris pour le cacaoyer. 
Tableau I.

Nombre de cacaoyères où une espèce est présente $(P)$, spontanée $(S)$ ou introduite $(I)$, sa fréquence $F(\%)$ et sa valeur d'usage relative (\%).

\begin{tabular}{|c|c|c|c|c|c|c|c|c|c|c|c|c|}
\hline \multirow[t]{2}{*}{ Espèce } & \multirow[t]{2}{*}{$\mathbf{P}$} & \multirow{2}{*}{$\mathrm{S}$} & \multirow{2}{*}{ I } & \multirow{2}{*}{$F(\%)$} & \multicolumn{7}{|c|}{ Valeur d'usage relative (V, \%) } & \multirow[b]{2}{*}{ U7 } \\
\hline & & & & & Totale & U1 & U2 & U3 & U4 & U5 & U6 & \\
\hline Albizia adianthifolia (Schumach.) W. Wight & 11 & 10 & 1 & 3,3 & 1,8 & - & - & 0,7 & 0,6 & 0,5 & - & - \\
\hline Albizia glaberrima (Schum. \& Thonn.) Benth. & 2 & 2 & - & 0,1 & 1 & - & - & 1 & - & - & - & - \\
\hline Alstonia boonei De Wild. & 10 & 10 & - & 1,9 & 2,8 & - & - & 0,5 & 1,4 & 0,5 & 0,4 & - \\
\hline Antrocaryon klaineanum Pierre & 4 & 4 & - & 0,9 & 1,2 & 0,2 & - & - & 0,8 & 0,2 & - & - \\
\hline Artocarpus altilis J.R. Forst. \& G. Forst. & 1 & 1 & - & 0,1 & 0 & - & - & - & - & - & - & - \\
\hline Berlinia confusa Hoyle & 2 & 2 & - & 0,1 & 1,5 & - & - & 0,5 & - & 1 & - & - \\
\hline Bombax buonopozense P. Beauv. & 1 & 1 & - & 0,1 & 0 & - & - & - & - & - & - & - \\
\hline Bridelia micrantha (Hochst.) Baill. & 5 & 5 & - & 0,5 & 1,2 & 0,4 & - & - & 0,8 & - & - & - \\
\hline Canarium schweinfurthii Engl. & 8 & 6 & 2 & 1,4 & 2 & 0,6 & - & - & 1 & 0,4 & - & - \\
\hline Carapa procera DC. & 1 & 1 & - & 0,1 & 0 & - & - & - & - & - & - & - \\
\hline Carpolobia alba G. Don & 2 & 2 & - & 0,1 & 0 & - & - & - & - & - & - & - \\
\hline Ceiba pentandra L. & 7 & 7 & - & 0,6 & 2,1 & - & - & - & - & 1,1 & 1 & - \\
\hline Citrus sinensis L. Osbeck & 4 & - & 4 & 0,5 & 5,7 & 3 & 2,2 & 0,2 & - & - & - & 0,3 \\
\hline Cleistopholis patens Benth. Engl. \& Diels & 3 & 3 & - & 0,4 & 0,3 & - & - & - & - & - & 0,3 & - \\
\hline Cola acuminata P. Beauv. Schott \& Endl. & 4 & 4 & - & 0,6 & 5 & 1,3 & 1,7 & - & - & 0,2 & 0,3 & 1,5 \\
\hline Cola ficifolia Mast. & 3 & 3 & - & 0,2 & 1,3 & 0,3 & 0,3 & - & - & 0,7 & - & - \\
\hline Cola lateritia K. Schum. & 4 & 4 & - & 0,7 & 1,3 & - & - & 0,5 & - & - & 0,8 & - \\
\hline Cola lepidota K. Schum. & 1 & 1 & - & 0,1 & 1 & 1 & - & - & - & - & - & - \\
\hline Cordia platythyrsa Baker & 6 & 6 & - & 1,2 & 1,7 & - & - & - & 1,5 & 0,2 & - & - \\
\hline Coula edulis Baill. & 5 & 5 & - & 0,9 & 3,4 & 0,4 & 0,8 & 0,4 & 1 & 0,6 & - & 0,2 \\
\hline Dacryodes edulis G. Don H. J. Lam & 14 & - & 14 & 17,7 & 6,4 & 3 & 1,7 & 0,2 & 0,8 & 0,3 & - & 0,4 \\
\hline Dacryodes macrophylla Oliv. H. J. Lam. & 5 & 2 & 3 & 1 & 4,6 & 1,4 & 0,8 & - & - & 0,8 & 0,8 & 0,8 \\
\hline Desbordesia glaucescens Engl. Van Tiegh. & 5 & 5 & - & 0,6 & 1,2 & - & - & - & - & 1,2 & - & - \\
\hline Discoglypremna caloneura Pax Prain & 5 & 5 & - & 1,5 & 2 & 1,2 & - & - & 0,8 & - & - & - \\
\hline Distemonanthus benthamianus Baill. & 7 & 7 & - & 0,9 & 3,9 & - & 0,3 & 2,6 & 1 & - & - & - \\
\hline Duboscia macrocarpa Bocq. & 1 & 1 & - & 0,2 & 0 & - & - & - & - & - & - & - \\
\hline Elaeis guineensis Jacq. & 9 & 2 & 7 & 2,2 & 6,4 & 2,4 & 2 & 0,3 & - & 0,9 & - & 0,8 \\
\hline Entandrophragma cylindricum Sprague Sprague & 5 & 5 & - & 0,8 & 6,8 & - & - & 1,8 & 1,8 & 2,2 & 1 & - \\
\hline Eribroma oblonga Mast. Pierre & 1 & 1 & - & 0,1 & 1 & - & - & 1 & - & - & - & - \\
\hline Erythrophleum ivorense A. Chev. & 1 & 1 & - & 0,2 & 0 & - & - & - & - & - & - & - \\
\hline Erythroxylon mannii Oliv. & 5 & 5 & - & 0,5 & 3,8 & - & 2,4 & 1,4 & - & - & - & - \\
\hline Fagara heitzii Aubrév. \& Pellegr. & 4 & 3 & 1 & 0,6 & 0,5 & - & - & 0,3 & - & - & 0,2 & - \\
\hline Ficus exasperata Valh & 10 & 10 & - & 2,7 & 2,8 & 0,2 & - & 0,3 & 0,4 & 1,4 & 0,3 & 0,2 \\
\hline Ficus mucoso Ficalho & 12 & 12 & - & 4,5 & 3,9 & - & - & 1,7 & 0,3 & 0,9 & 1 & - \\
\hline Ficus sur Forssk. & 3 & 3 & - & 0,7 & 0 & - & - & - & - & - & - & - \\
\hline Garcinia kola Heckel & 2 & 1 & 1 & 0,5 & 3,5 & 1,5 & 1 & - & - & 0,5 & - & 0,5 \\
\hline Glyphaea brevis Spreng. Monach. & 1 & 1 & - & 0,2 & 0 & - & - & - & - & - & - & - \\
\hline Guibourtia tessmannii & 1 & 1 & - & 0,1 & 2 & - & - & 1 & 1 & - & - & - \\
\hline Harungana madagascariensis Lam. ex Poir. & 1 & 1 & - & 0,1 & 0 & - & - & - & - & - & - & - \\
\hline Hevea brasiliensis A. Juss. Müll. Arg. & 1 & 1 & - & 0,1 & 0 & - & - & - & - & - & - & - \\
\hline Hylodendron gabunense Taub. & 2 & 2 & - & 0,1 & 0 & - & - & - & - & - & - & - \\
\hline Hypodaphnis zenkeri Engl. Stapf & 3 & 3 & - & 0,3 & 0,3 & - & - & - & 0,3 & - & - & - \\
\hline Irvingia gabonensis ex O’Rorke Baill. & 4 & - & 4 & 0,4 & 6 & 2,8 & 2 & - & 1,2 & - & - & - \\
\hline
\end{tabular}


Tableau I (suite).

\begin{tabular}{|c|c|c|c|c|c|c|c|c|c|c|c|c|}
\hline \multirow[t]{2}{*}{ Espèce } & \multirow[t]{2}{*}{$P$} & \multirow[t]{2}{*}{ S } & \multirow[t]{2}{*}{1} & \multirow[t]{2}{*}{$F(\%)$} & \multicolumn{7}{|c|}{ Valeur d'usage relative (V, \%) } & \multirow[b]{2}{*}{ U7 } \\
\hline & & & & & Totale & U1 & U2 & U3 & U4 & U5 & U6 & \\
\hline Klainedoxa gabonensis Pierre ex Engl. & 6 & 6 & - & 0,6 & 0,7 & - & - & - & 0,7 & - & - & - \\
\hline Lannea welwitschii Hiern Engl. & 3 & 3 & - & 0,4 & 0 & - & - & - & - & - & - & - \\
\hline Lovoa trichilioides Harms & 5 & 5 & - & 0,5 & 4 & - & 0,8 & 2,2 & - & 0,8 & - & 0,2 \\
\hline Macaranga hurifolia Beille & 5 & 5 & - & 1,2 & 1,4 & - & - & 1,4 & - & - & - & - \\
\hline Mangifera indica L. & 9 & - & 9 & 2,1 & 6,3 & 3 & 2,5 & - & 0,2 & 0,4 & - & 0,2 \\
\hline Markhamia lutea Benth. K. Schum. & 4 & 4 & - & 0,5 & 0 & - & - & - & - & - & - & - \\
\hline Massularia acuminata G. Don Bullock & 1 & 1 & - & 0,4 & 0 & - & - & - & - & - & - & - \\
\hline Milicia excelsa Welw. C.C. Berg. & 10 & 10 & - & 3,1 & 4,7 & - & 2,1 & 1,4 & 0,5 & 0,5 & 0,1 & 0,1 \\
\hline Millettia sp. & 1 & 1 & - & 0,1 & 0 & - & - & - & - & - & - & - \\
\hline Morinda lucida Benth. & 10 & 10 & - & 1,1 & 2,5 & - & - & - & 2,2 & 0,3 & - & - \\
\hline Musanga cecropioides R. Br. & 2 & 2 & - & 0,1 & 1 & - & - & - & - & 1 & - & - \\
\hline Myrianthus arboreus P. Beauv. & 2 & 2 & - & 0,3 & 1,5 & 1 & - & - & 0,5 & - & - & - \\
\hline Nauclea diderrichii De Wild. \& T.Durand merr. & 1 & 1 & - & 0,1 & 0,8 & - & - & 0,3 & 0,5 & - & - & - \\
\hline Newbouldia laevis Seem. ex Bureau & 6 & 6 & - & 0,9 & 2,4 & - & - & 1 & 0,7 & 0,7 & - & - \\
\hline Ongokea gore Hua Pierre & 3 & 3 & - & 0,4 & 0 & - & - & - & - & - & - & - \\
\hline Persea americana Mill. & 12 & - & 12 & 10,6 & 5,4 & 2,9 & 0,6 & - & 1,3 & 0,3 & - & 0,3 \\
\hline Petersianthus macrocarpus P. Beauv. Liben & 3 & 3 & - & 0,3 & 2,9 & 1 & 0,3 & 0,3 & - & 0,7 & 0,3 & 0,3 \\
\hline Phyllanthus discoideus Baill. Müll. Arg. & 8 & 8 & - & 2,4 & 0,8 & - & - & 0,6 & - & 0,2 & - & - \\
\hline Picralima nitida Stapf & 2 & 1 & 1 & 0,1 & 0 & - & - & - & - & - & - & - \\
\hline Piptadeniastrum africanum Hook. f. Brenan & 3 & 3 & - & 0,2 & 0 & - & - & - & - & - & - & - \\
\hline Polyalthia suaveolens Engl. \& Diels & 1 & 1 & - & 0,1 & 1 & - & - & 1 & - & - & - & - \\
\hline Porterandia cladantha K. Schum. Keay & 5 & 5 & - & 0,9 & 1,8 & - & - & 0,2 & 0,4 & 0,6 & - & 0,6 \\
\hline Pseudospondias microcarpa A. Rich. Engl. & 2 & 2 & - & 0,1 & 1 & - & - & - & - & 1 & - & - \\
\hline Pteleopsis hylodendron Mildb. & 4 & 4 & - & 0,3 & 0,7 & - & - & - & 0,5 & 0,2 & - & - \\
\hline Pterocarpus soyauxii Taub. & 11 & 11 & - & 1,9 & 3,4 & - & - & 1,3 & 1,4 & 0,7 & - & - \\
\hline Pycnanthus angolensis Welw. Warb & 10 & 10 & - & 2,4 & 3,9 & - & 0,2 & 0,7 & 1,4 & 1,2 & 0,4 & - \\
\hline Rauvolfia macrophylla Stapf & 3 & 3 & - & 0,3 & 5,6 & 2,3 & 3,3 & - & - & - & - & - \\
\hline Rauvolfia vomitoria Afzel. & 4 & 3 & 1 & 0,6 & 1,5 & - & - & - & 1 & 0,5 & - & - \\
\hline Ricinodendron heudelotii Baill. Pierre ex Heckel & 10 & 5 & 5 & 1,7 & 6 & 2,4 & 3,4 & - & 0,2 & - & - & - \\
\hline Schumanniophyton magnificum K. Schum. Harms & 1 & 1 & - & 0,1 & 0 & - & - & - & - & - & - & - \\
\hline Spathodea campanulata P. Beauv. & 10 & 10 & - & 1,9 & 1,9 & - & - & - & 1,3 & 0,6 & - & - \\
\hline Spondias cytherea Sonn. & 1 & - & 1 & 0,1 & 0 & - & - & - & - & - & - & - \\
\hline Staudtia kamerunensis Warb. & 4 & 4 & - & 0,4 & 3,5 & - & - & 2,2 & - & 1,3 & - & - \\
\hline Sterculia rhinopetala K. Schum. & 4 & 4 & - & 0,4 & 0 & - & - & - & - & - & - & - \\
\hline Terminalia superba Engl. \& Diels & 11 & 3 & 8 & 10,5 & 4 & - & 1,2 & 0,6 & - & 1,9 & - & 0,3 \\
\hline Tetrapleura tetraptera Schumach. \& Thonn. Taub. & 3 & 2 & 1 & 0,4 & 2,3 & 2,3 & - & - & - & - & - & - \\
\hline Tetrorchidium didymostemon Baill. Hoffm. & 1 & 1 & - & 0,1 & 1 & - & - & - & 1 & - & - & - \\
\hline Trichoscypha acuminata Engl. & 3 & 3 & - & 0,2 & 2 & 1 & - & - & - & - & - & 1 \\
\hline Triplochyton scleroxylon K. Schum. & 2 & 2 & - & 0,1 & 7,5 & - & - & 2,5 & - & 3 & 2 & - \\
\hline Tristemma mauritianum J. F. Gmel. & 2 & 2 & - & 0,3 & 0 & - & - & - & - & - & - & - \\
\hline Vernonia conferta Benth. & 4 & 4 & - & 0,4 & 1,8 & - & - & 0,5 & 1,3 & - & - & - \\
\hline Vitex grandifolia Gürke & 2 & 2 & - & 0,1 & 1,5 & - & - & 1,5 & - & - & - & - \\
\hline Xylopia aurantiodora De Wild \& T. Durand & 6 & 6 & - & 1,2 & 1,2 & 0,2 & - & 0,8 & - & 0,2 & - & - \\
\hline
\end{tabular}




\section{Usages des espèces}

Les enquêtes réalisées avec les agriculteurs lors des inventaires des espèces présentes dans leurs cacaoyères ont permis d'identifier sept usages ou services que nous avons regroupés en trois catégories : quatre usages pour la production, deux services écologiques, et un usage social (tableau II).

Parmi les espèces inventoriées dans les cacaoyères, 22 (25,5 \%) n'ont été affectées d'aucun usage, comme Artocarpus altilis (arbre à pain), Bombax buonopozense (kapokier) ou Erythrophleum ivorense (tali) ; et 14 espèces (16,3\%) n'ont eu qu'un seul usage (exemples : Eribroma oblonga et Albizia glaberrima). Les 50 autres espèces $(58,1 \%)$ ont eu entre deux et six usages, confirmant que la plupart des espèces sont multi-usages (figure 2). Les cinq espèces les plus fréquemment rencontrées dans les cacaoyères présentent ainsi trois usages, voire davantage : Dacryodes edulis (6 usages), Persea americana (5), Terminalia superba (4), Ficus mucoso (4), Albizia adianthifolia (3).

Les espèces inventoriées ont été réparties en fonction de leurs usages déclarés d'une part, et de la valeur que leur ont attribuée les agriculteurs pour chaque usage d'autre part (figure 3). La répartition de ces espèces par usage a été la suivante, par ordre décroissant : 59,4\% des espèces ont eu pour usage l'ombrage des cacaoyères, 54,7 \% la fourniture de produits médicinaux, 53,1\% la mise à disposition de produits ligneux destinés au ménage, 37,5\% la fourniture de produits non ligneux autoconsommés, 31,3\% l'offre de produits non ligneux et ligneux destinés à la vente, $25 \%$ l'usage social et $21,9 \%$ le maintien de la fertilité. La hiérarchie des usages a été la suivante : la valeur la plus élevée a été attribuée aux espèces destinées à la fourniture de produits destinés à la vente, qu'ils soient ligneux ou non ligneux $(47,6 \%)$; puis viennent les produits non ligneux autoconsommés et les produits ligneux destinés au ménage $(35,8 \%)$, les produits médicinaux (35,2\%), l’ombrage des cacaoyères $(29,7 \%)$, le maintien de la fertilité $(9,7 \%)$ et l'usage social (7,7\%).

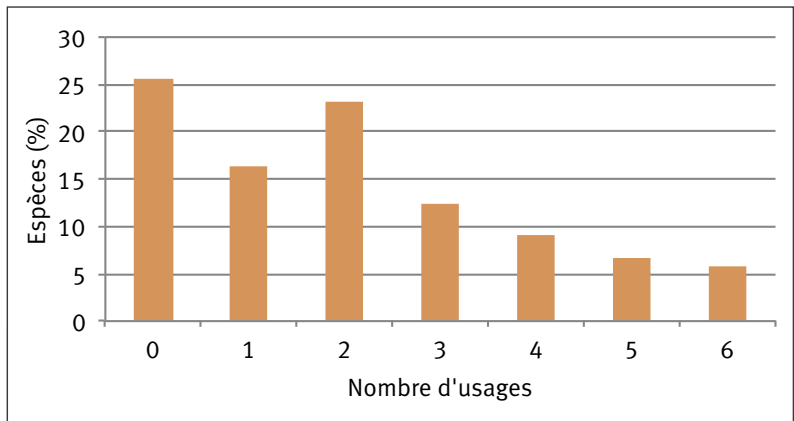

Figure 2.

Répartition des espèces inventoriées dans les cacaoyères agroforestières du Centre-Cameroun (\%) en fonction du nombre d'usages déclarés par les agriculteurs.
Tableau II.

Les sept usages ou services des espèces inventoriées dans les cacaoyères agroforestières du Centre-Cameroun.

\begin{tabular}{l|l|c} 
Catégorie & Usage ou service & Code \\
\multirow{2}{*}{ Production } & Produits non ligneux pour le ménage & U1 \\
\cline { 2 - 3 } & Produits ligneux et non ligneux pour la vente & U2 \\
\cline { 2 - 3 } & Produits ligneux pour le ménage & U3 \\
\cline { 2 - 3 } & Produits médicinaux pour le ménage & U4 \\
\hline Écologie & Ombrage des cacaoyers & U5 \\
\cline { 2 - 3 } & Maintien ou amélioration de la fertilité du sol & U6 \\
\hline Social & Produits non ligneux donnés ou offerts & U7 \\
\hline
\end{tabular}

\section{Classement des espèces selon leur valeur d'usage}

L'espèce qui a eu la valeur d'usage la plus élevée pour les agriculteurs, soit 19,6\%, a été le cacaoyer, Theobroma cacao, confirmant ainsi le rôle pivot de cette espèce dans le système agroforestier. La valeur d'usage la plus élevée attribuée à $T$. cacao a concerné la production de cacao pour la vente (18\%). Les agriculteurs ont également attribué une valeur d'usage à cette espèce pour la fourniture de produits médicinaux $(0,9 \%)$, le maintien de la fertilité du sol $(0,2 \%)$ et la fourniture de bois $(0,1 \%)$.

La valeur attribuée aux autres espèces est présentée dans le tableau I. Pour l'ensemble des 14 cacaoyères agroforestières, après le cacaoyer et par ordre décroissant, les 10 espèces qui ont eu les valeurs d'usage les plus élevées ont été l'ayous, Triplochyton scleroxylon (7,5\%), le sapelli, Entandrophragma cylindricum (6,8 \%), le safoutier (6,4 \%), le palmier à huile $(6,4 \%)$, le manguier, Mangifera indica $\mathrm{L}$. (6,3\%), l'avocatier (5,4\%), le manguier sauvage, Irvingia gabonensis (6\%), le njangsang, Ricinodendron heudelotii (6\%), l'oranger, Citrus sinensis (5,7\%), et le Mejan Mejanga, Rauvolfia macrophylla (5,6 \%).

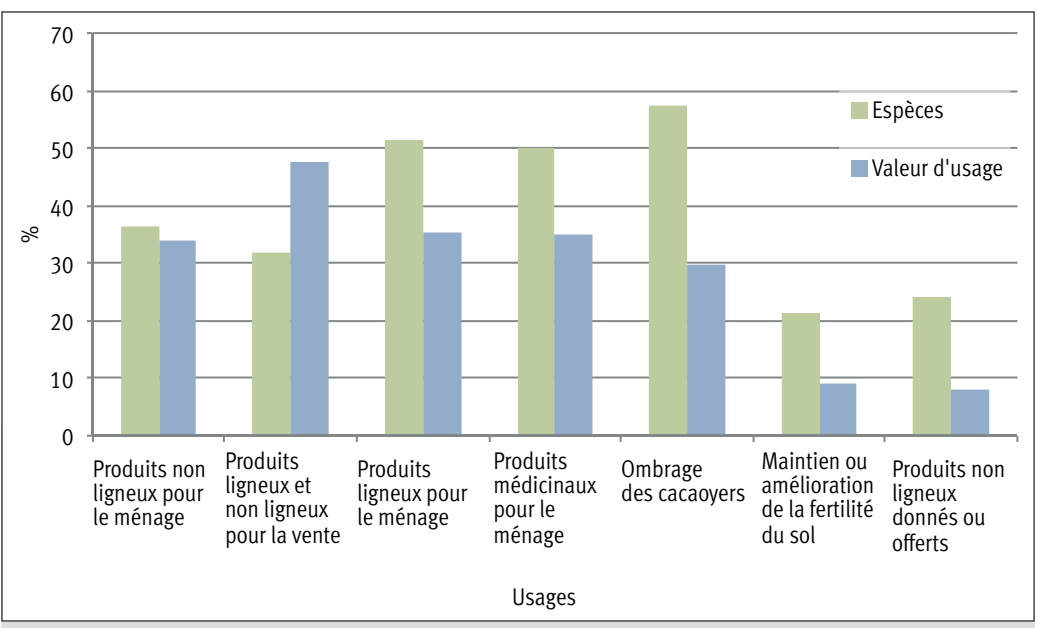

Figure 3.

Espèces inventoriées dans les cacaoyères agroforestières du Centre-Cameroun, y compris le cacaoyer (\%), et valeur d'usage totale (\%) pour chacun des sept usages et services définis par les agriculteurs. 


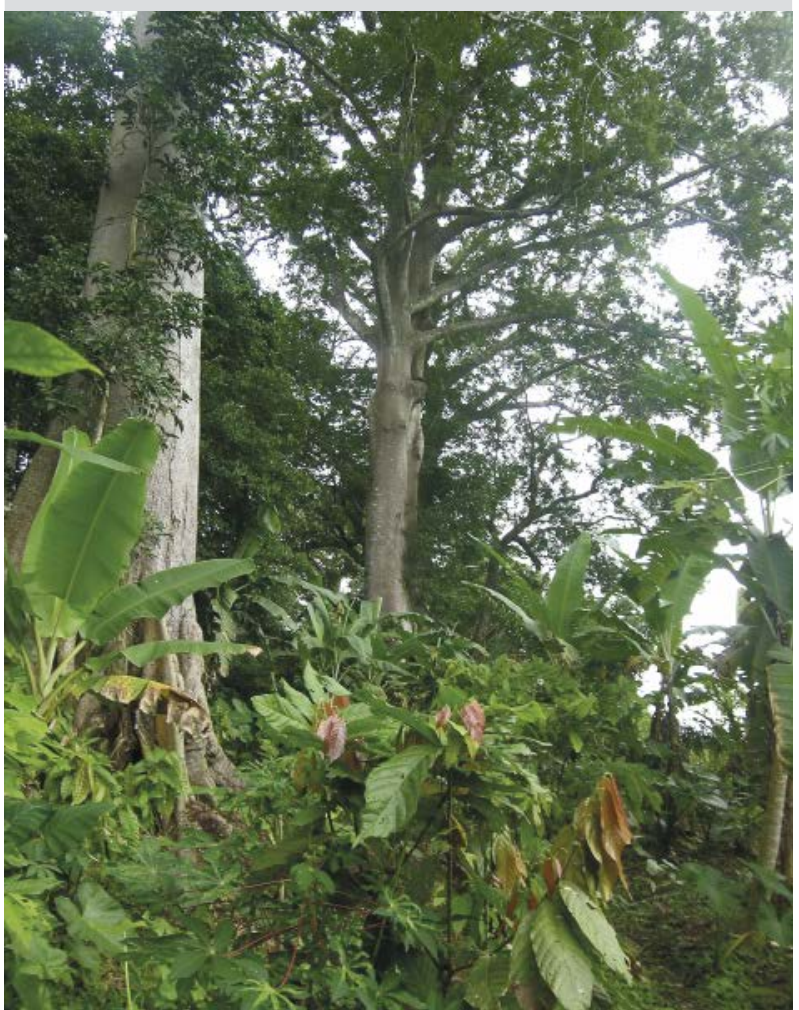

La méthode participative que Sheil et al. (2004) ont conçue pour connaître la valeur accordée par certaines communautés villageoises indonésiennes à leur milieu et à leurs ressources naturelles a permis d'estimer la valeur relative que les agriculteurs du Centre-Cameroun attribuent aux différentes espèces présentes dans leurs cacaoyères agroforestières. Cette méthode a présenté plusieurs atouts. Similaire à la méthode inspirée du jeu du Bao (Franzel, 2001), la méthode de distribution des cailloux permet de combiner la collecte de données quantitatives, analysables statistiquement, à une évaluation participative où les agriculteurs s'impliquent et justifient leurs choix. Elle a par ailleurs été facile à mettre en œuvre car aisément compréhensible par les agriculteurs. Les intitulés des colonnes et des lignes du tableau croisé, ici les noms vernaculaires des espèces et des usages, peuvent être complétés par des icônes ou des photos. Un autre avantage de cette méthode est de pouvoir relier des observations facilement mesurables, comme la fréquence des espèces, à la valeur d'usage, dans le but d'identifier les espèces qui, outre le cacaoyer, constituent le socle des systèmes agroforestiers analysés. Enfin, le classement des espèces réalisé par les agriculteurs, pondéré par leurs usages, donne accès à un niveau d'information supérieur à celui des méthodes habituellement utilisées pour connaître le savoir-faire écologique traditionnel, telles que les interviews, questionnaires, ateliers (Martin et

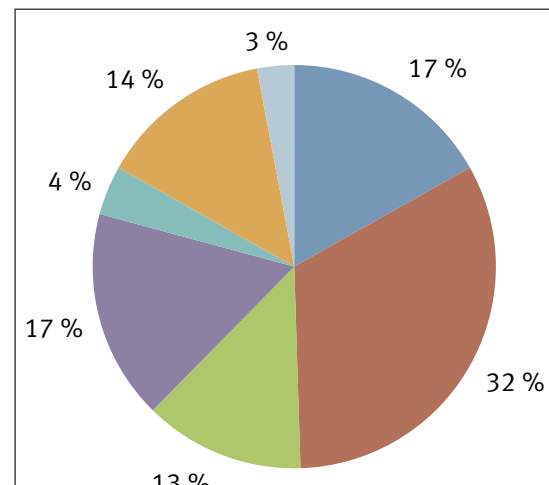

Produits non ligneux
pour le ménage
Produits ligneux et non
ligneux pour la vente
Produits ligneux pour
le ménage
Produits médicinaux
pour le ménage
Ombrage des cacaoyers
Maintien ou amélioration
de la fertilité du sol
Produits non ligneux
donnés ou offerts

Photo 4.

Ceiba pentandra est, avec Ficus mucoso, l'une des principales espèces conservées par les agriculteurs pour maintenir la fertilité des sols sous cacaoyers. Photo P. Jagoret.

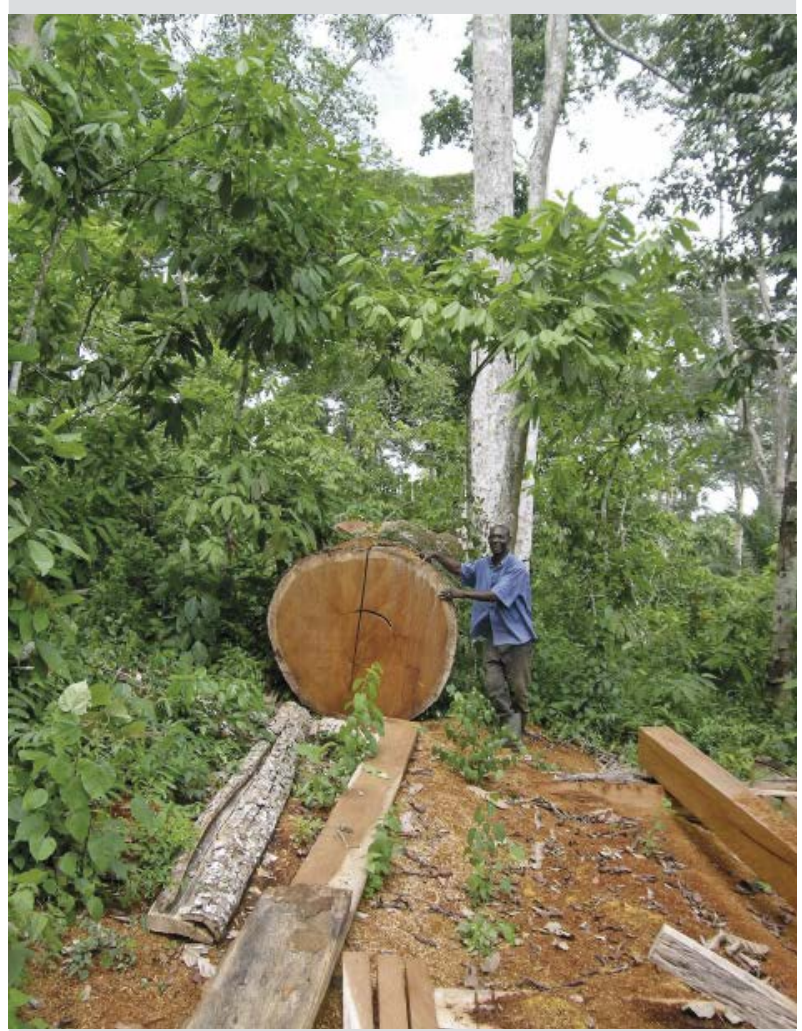

Photo 5.

La fourniture de bois, qui représente $13 \%$ de la valeur totale attribuée par les agriculteurs aux différentes espèces, est le quatrième usage dévolu aux cacaoyères agroforestières en termes de valeur. Photo P. Jagoret.

Figure 4.

Profil d'usage des cacaoyères agroforestières du Centre-Cameroun. 
al., 2010). En tant que méthode holistique qui pondère la valeur d'usage d'une espèce par rapport aux autres, évaluer la valeur d'usage des espèces prend en compte l'intégralité des espèces qui constituent les systèmes agroforestiers et l'ensemble de leurs usages.

En termes de densité, le peuplement cacaoyer est la composante principale des systèmes agroforestiers du Centre-Cameroun (Jagoret et al., 2011). En attribuant la valeur d'usage la plus élevée au cacaoyer, les agriculteurs confirment ainsi que cette espèce, qui est leur principale source de revenus (Leplaideur, 1985 ; Jagoret et al., 2011), constitue le pivot de ces systèmes.

Il serait toutefois intéressant d'évaluer l'impact de la fluctuation du prix d'achat du cacao, et des autres produits issus du système (fruits, bois, etc.) sur la valeur d'usage qu'attribueraient les agriculteurs au cacaoyer et aux autres espèces. En 2009, le prix d'achat du cacao marchand au producteur était en effet de l'ordre de 700 FCFA/kg (N.B. : 656 FCFA = $1 €)$, alors qu'il a fortement augmenté en 2011 pour atteindre 1500 FCFA/kg. On peut ainsi faire l'hypothèse que la valeur d'usage relative de Theobroma cacao augmente avec le prix d'achat du cacao, au détriment de la valeur accordée aux autres espèces ayant un intérêt commercial, alors que ce pourrait être l'inverse dans le cas d'une chute du prix du cacao.

À Ngomedzap, les espèces fruitières fournissent principalement des produits destinés à la consommation familiale et à la vente et jouent aussi un rôle social, alors que les espèces forestières couvrent les autres usages : bois de chauffe et bois d'œuvre, produits médicinaux, ombrage des cacaoyères, maintien de la fertilité du sol. Reproduire la même évaluation dans une autre zone que la zone forestière, comme la zone de transition forêt-savane ou le bassin de production du Sud-Ouest, aux conditions pédoclimatiques contrastées, permettrait cependant de vérifier l'impact de ces conditions sur l'utilisation des espèces associées aux cacaoyers par les agriculteurs et leur valeur. Dans la zone de transition forêt-savane où le nombre d'espèces forestières est largement moindre qu'en zone forestière, il est ainsi possible que certains usages dévolus à ces dernières soient assurés par les espèces fruitières, plus fréquentes dans les cacaoyères qu'en zone forestière (Jagoret et al., 2012).

En tant que plante médicinale, le cacaoyer est utilisé principalement comme antiseptique local (jeunes feuilles utilisées pour désinfecter les plaies). Zapfack et al. (2002) avaient déjà signalé un usage médicinal du cacaoyer dans les zones forestières du Centre-Cameroun, sans toutefois préciser la teneur de cet usage. Parmi les cinq espèces les mieux classées par les agriculteurs dans l'usage de maintien de la fertilité du sol, Ficus mucoso, Ceiba pentandra et Entandrophragma cylindrica sont également présentes dans le classement de Bidzanga et al. (2009) dans la même zone. Ces auteurs, après avoir demandé à 20 agriculteurs de classer 10 espèces "fertilitaires » inventoriées dans leurs cacaoyères, ont mis en évidence que le classement de ces dernières était similaire à leur classement basé sur le taux de colonisation des racines de ces espèces par les mycorhizes.

L'évaluation participative de la valeur d'usage des espèces a permis d'identifier quelles étaient les principales espèces des systèmes agroforestiers du Centre-Cameroun. L'importance pour les agriculteurs d'espèces fruitières comme

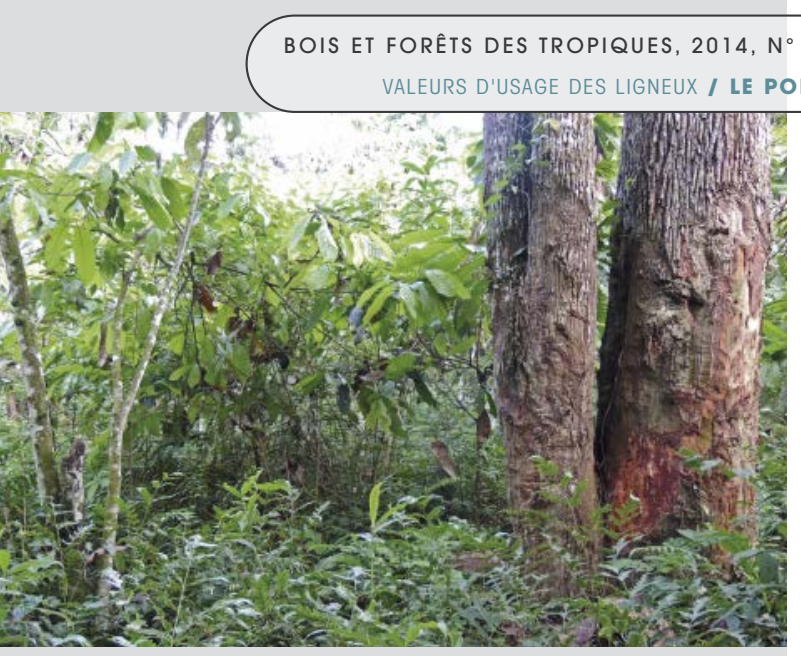

Photo 6.

Alstonia boonei (ekuk en ewondo), dont l'écorce est un antipaludéen, est une espèce médicinale présente dans la plupart des cacaoyères du Centre-Cameroun. Photo P. Jagoret.

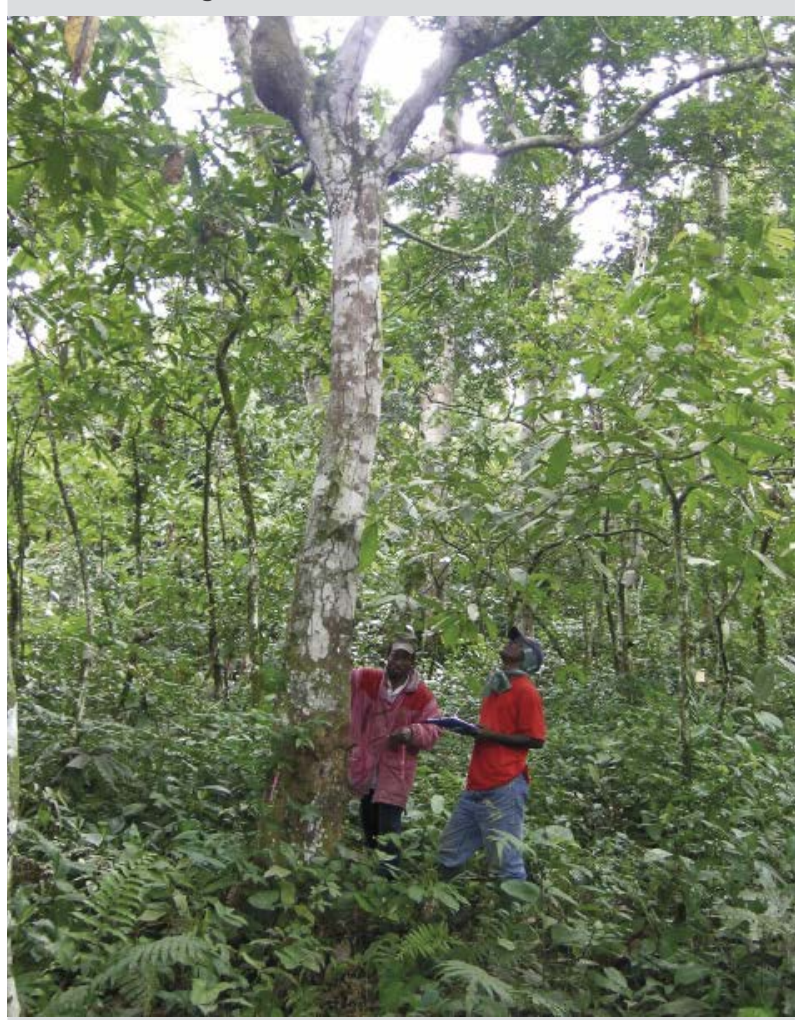

Photo 7.

L'évaluation de la valeur d'usage des cacaoyères agroforestières passe par un inventaire préalable des différentes espèces ligneuses associées aux cacaoyers. Photo P. Jagoret.

Dacryodes edulis, Elaeis guineensis, Persea americana et Citrus sinensis pour la production de fruits pour la consommation et la vente est confirmée. Toutefois, parmi les espèces qui constituent le socle des systèmes agroforestiers cacaoyers, plusieurs espèces forestières figurent comme Terminalia superba ou Ficus mucoso dont les usages sont différents des espèces fruitières : bois d'œuvre, fertilité du sol, ombrage des cacaoyers. Si l'introduction d'espèces à fort potentiel économique constitue la principale voie pour améliorer les systèmes agroforestiers cacaoyers (Ruiz Pérez et al., 1999 ; Leakey et Tchoundjeu, 2001 ; Schreckenberg et al., 2006), les résultats 
obtenus montrent que d'autres possibilités doivent être envisagées. Les attentes des agriculteurs vont en effet au-delà de la fourniture de produits pour la consommation et la vente, et les autres usages que les espèces assurent doivent être considérés. Cela est confirmé par le profil d'usage des cacaoyères agroforestières où les usages autres que la fourniture de produits pour la consommation et la vente représentent plus de $50 \%$ de la valeur d'usage totale attribuée par les agriculteurs en mobilisant la méthode de distribution des cailloux.

\section{Conclusion}

La majorité des espèces présentes dans les cacaoyères agroforestières du Centre-Cameroun a une valeur explicite pour les agriculteurs. La plurifonctionnalité des cacaoyères agroforestières se décline à la fois à l'échelle de la parcelle et à celle de l'espèce car nombreuses sont les espèces qui répondent aux besoins du cacaoyer ainsi qu'à ceux des ménages agricoles. Les voies d'amélioration des systèmes agroforestiers cacaoyers doivent donc tenir compte de la valeur d'usage accordée aux espèces ligneuses par les agriculteurs afin de leur proposer des types d'associations cacaoyer/espèces ligneuses répondant mieux à leurs attentes et d'assurer ainsi une meilleure adoption des innovations techniques.

\section{Remerciements}

Les travaux sur les systèmes agroforestiers cacaoyers du Centre-Cameroun ont été conduits dans le cadre du dispositif de recherche et d'enseignement en partenariat "Agroforesterie Cameroun », en partenariat avec l'Institut de recherche agricole pour le développement (Irad) et le Centre de coopération internationale en recherche agronomique pour le développement (Cirad). Ils ont été entrepris dans le cadre du « Projet de renforcement des partenariats dans la recherche agronomique au Cameroun », financé par le Ministère des Affaires étrangères et du Développement international (France).

\section{Références bibliographiques}

BIDZANGA N., FOTSING B., AGOUME V., BIRANG A. M., ONGUENE A. N., ZAPFACK L., 2009. Mycotrophie et connaissances paysannes des essences fertilitaires dans les agroforêts à base de cacaoyers du Sud Cameroun. Cameroon Journal of Experimental Biology, 5 : 79-86.

BISSELEUA D. H. B., MISSOUP A. D., VIDAL S., 2009. Biodiversity conservation, ecosystem functioning, and economic incentives under cocoa agroforestry intensification. Conservation Biology, 23 (5): 1176-1184.

EYOG MATIG O., NDOYE O., KENGUE J., AWONO A., 2006. Les fruitiers forestiers comestibles du Cameroun. Cotonou, Bénin, IPGRI/Saforgen/Irad/Cifor, 204 p.

FRANZEL S., 2001. Use of an indigenous board game, "Bao”, for assessing farmers' preferences among alternative agricultural technologies. In: Peters G. H., Pingali P. (eds). Tomorrow's agriculture: incentives, institutions, infrastructure and innovations. Oxford, Royaume-Uni, Ashgate, 416-424.
HUXLEY P. A., 1999. Tropical Agroforestry. Londres, RoyaumeUni, Blackwell Science, $371 \mathrm{p}$.

ICCO, 2012. The world cocoa economy: past and present. Londres, Royaume-Uni, ICCO, $42 \mathrm{p}$.

JAGORET P., MICHEL-DOUNIAS I., MALÉZIEUX E., 2011. Longterm dynamics of cocoa agroforests: a case study in central Cameroon. Agroforestry Systems, 81 (3): 267-278.

JAGORET P., MICHEL-DOUNIAS I., SNOECK D., TODEM NGNOGUE H., MALÉZIEUX E., 2012. Afforestation of savannah with cocoa agroforestry systems: a small-farmer innovation in central Cameroon. Agroforestry Systems, 86: 493-504.

LEAKEY R. R. B., TCHOUNDJEU Z., 2001. Diversification of tree crops: domestication of companion crops for poverty reduction and environmental services. Experimental Agriculture, 37 (3): 279-296.

LEPLAIDEUR A., 1985. Les systèmes agricoles en zone forestière : les paysans du Centre et du Sud Cameroun. Paris, France, Cirad-Irat, $615 \mathrm{p}$.

MARTIN J. F., ROY E. D., DIEMONT S. A. W., FERGUSON B. G., 2010. Traditional Ecological Knowledge (TEK): Ideas, inspiration, and designs for ecological engineering. Ecological Engineering, 36 (7): 839-849.

NAIR P. K. R., 1993. An introduction to agroforestry. Dordrecht, Pays-Bas, Kluwer Academic Publishers, Nairobi, Kenya, Icraf, 292 p.

RUF F., SCHROTH G., 2004. Chocolate forests and monocultures: A historical review of cocoa growing and its conflicting role in tropical deforestation and forest conservation. In: Schroth G., Da Fonseca G. A. B., Harvey C. A., Gascon C., Vasconcelos H. L., Izac A. M. N. (eds). Agroforestry and biodiversity conservation in tropical landscapes Washington, États-Unis, Island Press, 107-133.

RUIZ PÉREZ M., NDOYE O., EYEBE A., 1999. Marketing of non-wood forest products in the humid forest zone of Cameroon. Unasylva, 198: 12-19.

SANTOIR C., BOPDA A., 1995. Atlas régional Sud-Cameroun. Paris, France, Orstom, 53 p.

SHEIL D., PURI R. K., BASUKI I., VAN HEIST M., WAN M., LISWANTI N., RUKMIYATI, SARDJONO M. A., SAMSOEDIN I., SIDIYASA K., CHRISANDINI., PERMANA E., ANGI E. M., GATZWEILER F., JOHNSON B., WIJAYA A., 2004. À la découverte de la biodiversité, de l'environnement et des perspectives des populations locales dans les paysages forestiers. Méthodes pour une étude pluridisciplinaire du paysage. Jakarta, Indonésie, Cifor, $97 \mathrm{p}$.

SCHRECKENBERG K., AWONO A., DEGRANDE A., MBOSSO C., NDOYE O., TCHOUNDJEU Z., 2006. Domesticating indigenous fruit trees to reduce poverty. Forest Trees Livelihoods, 16: 35-51.

SONWA D. J., NKONGMENECK A. B., WEISE S. F., TCHATAT M., ADESINA A. A., JANSSENS M. J., 2007. Diversity of plants in cocoa agroforests in the humid forest zone of Southern Cameroon. Biodiversity and Conservation, 16 (8): 2385-2400. TORQUEBIAU E. F., MARY F., SIBELET N., 2002. Les associations agroforestières et leurs multiples enjeux. Bois et Forêts des Tropiques, $271:$ 23-35.

VIVIEN J., FAURE J.-J., 1985. Arbres des forêts denses d'Afrique centrale. Espèces du Cameroun. Paris, France, Agence de coopération culturelle et technique, $565 \mathrm{p}$.

ZAPFACK L., ENGWALD S., SONKE B., ACHOUNDONG G., BIRANG A. M., 2002. The impact of land conversion on plant biodiversity in the forest zone of Cameroon. Biodiversity and Conservation, 11 (11): 2047-2061. 